\title{
Applying mathematical equations and computer programming to obtain transferred energy into body tissue
}

\begin{abstract}
In this paper, a mathematical method is investigated for therapy by fast neutron. This method can be applied for all tissues and investigations in connection with cancer therapy. The mathematical method is considered using equations and computer programming. The amounts of Kerma are calculated by this mathematical method in a liver tissue for a wide range of energies as a result of collision between neutrons with nuclei of constituent elements of tissue that have various mass numbers. This method can be applied to estimate the energy of neutrons reached the appropriate energy range through deducing the incident neutron energy emitted from clinical neutron source for a wide spectrum of neutron energies.
\end{abstract}

Volume I Issue 4 - 2018

Mousavi Shirazi SA

Department of Physics, Islamic Azad University, Iran

Correspondence: Mousavi Shirazi SA, Department of Physics, Islamic Azad University, Iran, Tel: +989I 220270 59,

Email alireza_moosavi@yahoo.com, a_moosavi@azad.ac.ir

Received: June 15, 2018 | Published: July 27, 2018

Keywords: kerma, mathematical equations, neutron, programming, therapy

\section{Introduction}

Radiation therapy is a technique that has been designed to treat tumors through high LET heavy charged particles radiation at the cellular level. The explanation of the unusually large thermal neutron capture cross section for natural isotope ${ }^{10} \mathrm{~B}$ by Goldhaber was performed in 1934 . He explored that ${ }^{10} \mathrm{~B}$ is having an unusually high willingness to absorb either slow or thermal neutrons (for $\mathrm{E}<0.1 \mathrm{eV}$ ). It is considered that reactor based epithermal neutron beams with near optimum characteristics are currently available, and more can be constructed at existing reactors. The suitable reactors those which include low power reactors generally, using the core as a source of neutrons and also existing moderator might be used for radiation therapy, if the mono energy neutron source is not accessible. ${ }^{1,2}$ The boric acid solution moderator might be suitable for the spectrum measurement of an epithermal neutron irradiation field. Thus, computation and modeling of the delivered energy before practical treatment is also recommended. ${ }^{3}$ In this research, there are two goals. First of all is calculation of the amount of Kerma in the liver tissue within the course of radiation therapy by neutron. ${ }^{4}$ The second objective is to find out a way to reach eligible energy range of neutrons according to acceptable dose in radiation therapy and also studying the deduction of incident neutron energy emitted from neutron source for a wide spectrum of neutrons in a way that it can obtain the accurate amounts of Kerma and neutron energy reached components of a tissue during the therapy, and subsequently obtaining the required irradiation time for radiation therapy. ${ }^{5,6}$ The different forms of tissues like cubic format for body parts of human in some research have already been applied. ${ }^{7}$ A liver tissue includes substances such as water, glycogen and heavy molecules like protein and glucose. But in general, in such kind of research, the main aim is to simulate the nuclear and atomic interactions in the material. ${ }^{8,9}$

\section{Materials and methods}

\section{Applying mathematical method}

The advantage of the adopted analytical method is application of a method except usual methods like Monte Carlo simulation so that this method is based on diffusion equation instead of transport equation which MCNP code uses. Neutrons are emitted from a neutron source, and pass through carbon, and are slowed down. There is the analytical method scrutinized to find out the behavior of neutron interactions in tissue considering effectiveness of the neutron collision in various energies, thereby the absorbed energy is computed by analytical computations. In this method, passing the neutrons through the cited tissue and also having the knowledge about neutron angular distribution after scattering are actually essential. There are three major types of interactions with carbon and hydrogen nuclei. These are elastic scattering, inelastic scattering, and radioactive capture. ${ }^{10}$ Collision of neutrons on carbon and hydrogen nuclei causes some energy to transfer from neutrons to the target nuclei.

Two of neutron collision systems are according to Figures 1-3.

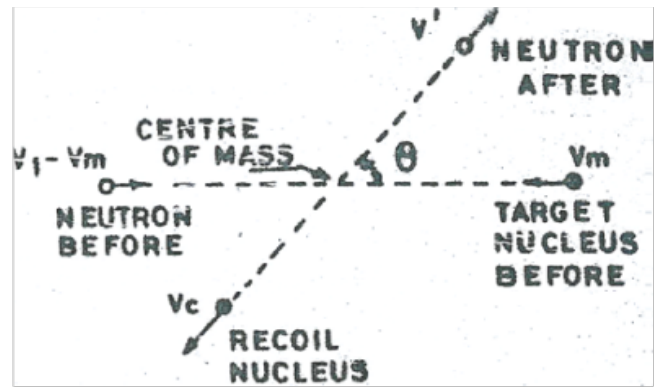

Figure I Centre of Mass (CM) system.

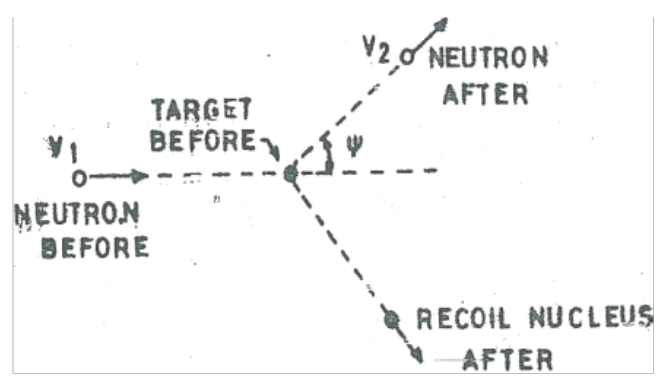

Figure 2 Laboratory system. 


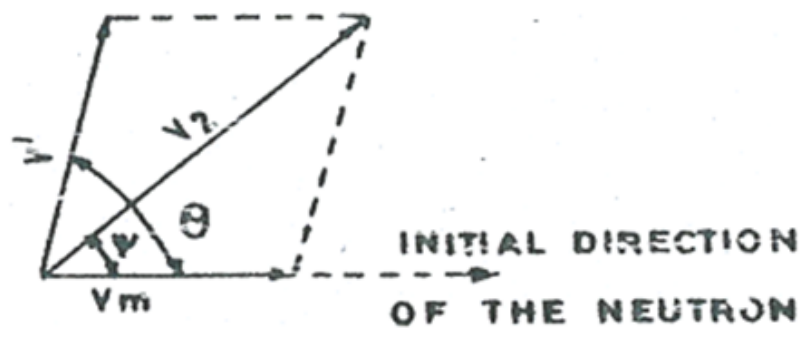

Figure 3 Conversion of CM system into the laboratory system.

There can be two following equations written:

$$
\begin{aligned}
& v_{2}^{2}=v_{1}^{2} \frac{A^{2}+2 A \operatorname{Cos} \theta+1}{(A+1)^{2}} \\
& \frac{v_{2}^{2}}{v_{1}^{2}}=\frac{E_{2}}{E_{1}}=\frac{A^{2}+2 A \operatorname{Cos} \theta+1}{(A+1)^{2}}
\end{aligned}
$$

Where:

$E_{1}$ : initial energy of neutron before interaction

$E_{2}$ : final energy of neutron after interaction

A: atomic weight of recoiled nucleolus resulting from neutron collision

$\theta$ : The angle between target nucleolus path and recoiled neutron in the center of mass system

And also:

$$
\frac{E_{2}}{E_{1}}=\frac{1}{2}[(1+\alpha)+(1-\alpha) \operatorname{Cos} \theta]
$$

Where:

$$
\alpha=\left(\frac{A-1}{A+1}\right)^{2}, i f: A=1 \rightarrow \alpha=0
$$

If the $v_{i 1}$ and $v_{i 2}$ are the initial velocities of proton and neutron respectively, then the $v_{f 1}$ and $v_{f 2}$, which are the final velocities, will be as following equation (Eq.5):

$$
\left\{\begin{array}{l}
v_{f 1}=\frac{m_{1}-m_{2}}{m_{1}+m_{2}} v_{i 1}+\frac{2 m_{2}}{m_{1}+m_{2}} v_{i 2} \\
v_{f 2}=\frac{2 m_{1}}{m_{1}+m_{2}} v_{i 1}+\frac{m_{2}-m_{1}}{m_{1}+m_{2}} v_{i 2}
\end{array}\right.
$$

After collision at 0 degree angle and head to head collision and also having been recoiled of the proton, there will be the final velocity of proton and neutron respectively as below:

$$
\begin{aligned}
& v_{f 1}=v_{i 2} \text { and } v_{f 2}=v_{i 1} \\
& E_{p}=\frac{1}{2} m_{n} v_{i 1}^{2} \text { and } E_{p}=\frac{1}{2} m_{p} v_{f 2}^{2}
\end{aligned}
$$

The recoiled nucleus moves a short distance through the matter, and deposits its energy along the path. The problem is to compute the energy of recoiled nucleus, and it involves applying the neutron diffusion equation. ${ }^{11,12}$

Therefore, the maximum rate of $\frac{E_{2}}{E_{1}}$ (or on the other hand

minimum lost energy) occurs whenever $\theta=0$ and the maximum lost energy will occur in the case of $\theta=\pi$.

$\theta$ : collision angle of incident neutron

The tissues which are in this path are: adipose tissue and skin tissue. One of the most important tissues, which sets in front of the liver, is adipose tissue.

The high LET includes protons resulting from the capture reaction of thermal neutrons with nitrogen atoms $\left[{ }^{14} \mathrm{~N}(n, p){ }^{14} \mathrm{C}\right]$ and also recoiled protons resulting from the collision of fast neutrons with hydrogen atoms. In addition, the high LET of protons is produced through fast neutrons scattering. The low LET of gamma rays is resulting from the capture of thermal neutrons with tissue and also hydrogen atoms $\left[{ }^{1} \mathrm{H}(\mathrm{n}, \gamma)^{2} \mathrm{H}\right]{ }^{13}$

In the laboratory system, because of having collision between a neutron and a nucleus, the amount of energy transferred to a recoiled nucleus is computed with Eq.8:11

$$
E_{R}=E_{1}-\left(E_{2}+E_{x}\right)
$$

The energy and scattering angle in inelastic scattering are obtained as following equations (Eqs.9-11):

$$
\begin{gathered}
E_{2}=\left(\frac{A}{A+1}\right)^{2} E_{1}+E^{\prime}+\frac{2 \operatorname{Cos} \theta}{(A+1)} \sqrt{E_{1} E^{\prime}} \\
\operatorname{Cos} \psi=\frac{1}{\sqrt{E_{2}}}\left[\frac{A}{A+1} \sqrt{E_{1}}+\sqrt{E^{\prime}} \operatorname{Cos} \theta\right] \\
E^{\prime}=\frac{A}{A+1}\left(\frac{A}{A+1} \cdot E_{1}-E_{x}\right)
\end{gathered}
$$

Where:

$E_{R}:$ transferred energy

$E_{x}:$ the excitation energy in recoiled nuclei

$E^{\prime}$ : scattered neutron energy in the centre of mass system

$\psi$ : the angle between the path of recoiled nucleus and incident neutron in the laboratory system

For high neutron energies, the reaction of neutron and alpha plays an important role among all the reactions in a way that in this state, some part of Kerma is because of alpha particles. In this case, the calculation is performed by neutron angular distribution after scattering and random sampling of neutron collision in either elastic or inelastic scattering. The atomic composition of the soft tissue is approximated with hydrocarbon materials. In this investigation, neutron slowing down has been taken into account as well. Therefore, according to Eq.8, the amount of dose in the tissue is obtained. In order to reach the required absorbed dose according to energy of neutron source, using the Eqs.8-11, the amount of dose in the mentioned tissue is obtained. The Eq. 10 is defined based on calculation of $\cos \theta$ and generation of random numbers. Finally, the irradiation time can be obtained to reach the eligible KERMA in a way that it might be applied for a patient who sets under radiation therapy by neutron.

\section{Results}

The amount of Kerma is calculated because of emitting a neutron beam with constituent elements of tissue having various mass numbers (for a wide range) per the mass number of constituent elements of it. The amount of Kerma is calculated in the mentioned liver tissue. The derived results are illustrated in Figure 4 using mathematical 
equations and computer programming for wide neutron energy.

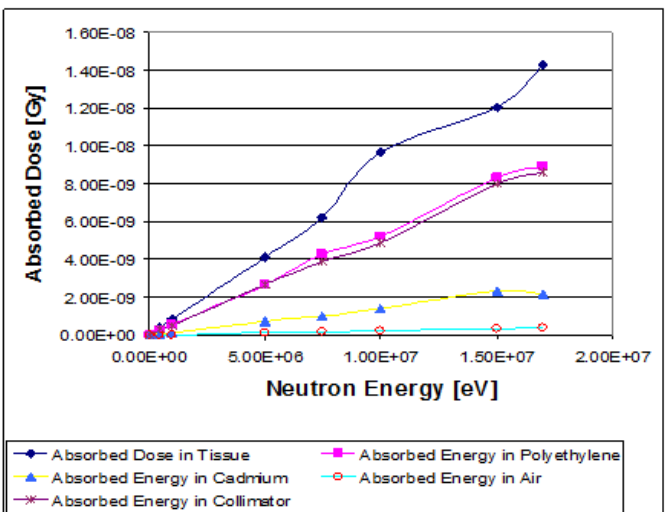

Figure 4 Amounts of kerma in the compounds of tissue for a wide range of energies.

\section{Conclusion}

The mathematical method provides dose calculation based on mathematical equations and considering $\psi$ and neutron energies despite being carried out the calculation based on neutron tracking and transport equation in some of nuclear codes like MCNP code. By the mentioned method, it can be inferred that how much time as the required irradiation time will take as long as the eligible dose is reached for treating liver cancer by radiation therapy for every one of liver tissues which have various dimensions and components. Therefore for each patient, given the desired dose for therapy and also specifications of neutron source, the required irradiation time for similar liver tissues can also be obtained. As it is observed in Figure 4, the maximum Kerma arises for elements having small mass number like hydrogen. It means the most amounts of absorbed dose Kerma are related to liver tissue because of abundance of hydrogen in it. This figure implies that with higher neutron energy, more doses may be delivered to the liver tissue.

\section{Acknowledgments}

None.

\section{Conflict of interest}

The author declares that there is no conflict of interest.

\section{References}

1. Rafiei Karahroudi M, Mousavi Shirazi SA. Study of power distribution in the CZP, HFP and normal operation states of VVER-1000 (Bushehr) nuclear reactor core by coupling nuclear codes. Ann Nucl Energy. $2015 ; 75: 38-43$

2. Rafiei Karahroudi M, Mousavi Shirazi SA. Obtaining the neutronic and thermal hydraulic parameters of the VVER-1000 Bushehr nuclear reactor core by coupling nuclear codes. Kerntechnik. 2014;79(6):528-531.

3. Mousavi Shirazi SA. Numerical solution of diffusion equation to study fast neutrons flux distribution for variant radii of nuclear fuel pin and moderator regions. Kerntechnik. 2015;80(3):291-294.

4. Mansour M, Warad I, Saffarini G, et al. Evaluation of U238 and Th232 radionuclide activities in kidney gallstone belonging to cancer patient compared with normal one by $\mathrm{X}$-ray spectrometry and EDS. $J$ Mater Environ Sci. 2015;6(10):2717-2721.

5. Mousavi Shirazi SA, Rastayesh S. The comparative investigation and calculation of thermo-neutronic parameters on two gens II and III nuclear reactors with same powers. WASET. 2011;5:99-103.

6. Faiz Z, Fakhi S, Bouih A, et al. Radioactive waste management: optimization of the mechanical property of cemented Ion Exchange Resin. J Mater Environ Sci. 2012;3(6):1129-1136.

7. Lamchouri F, Toufik H, Bouzzine SM, et al. Experimental and computational study of biological activities of alkaloids isolated from Peganum harmala seeds. J Mater Environ Sci. 2010;1:343-352.

8. Sheibani J, Mousavi Shirazi SA, Rahimi MF. Studying the effects of compound nucleus energy on coefficient of surface tension in fusion reactions using proximity potential formalism. J Fusion Energ. 2014;33(1):74-82.

9. Annals of the ICRP Recommendations of the International Commission Radiological Protection(ICRP). New York. 1977;1(3).

10. Andrieux ML, Dinkespiler B, Lundquist J, et al. Neutron and gamma irradiation studies of packaged VCSEL emitters for the optical read-out of the ATLAS electromagnetic calorimeter. Nuc Instr Meth Phys Res. 1999;426(2-3):332-338.

11. Bolewski AJ, Ciechanowski M, Dydejczyk A, et al. On the optimization of the isotopic neutron source method for measuring the thermal neutron absorption cross section: Advantages and disadvantages of $\mathrm{BF}_{3}$ and ${ }^{3} \mathrm{He}$ counters. Appl Rad Isot. 2008;66(4):457-462.

12. Dhairyawan M, Nagarajan P, Venkataraman G. Response functions of spherically moderated neutron detectors. Nuc Instr Meth. 1980;169(1):115-120.

13. Coderre J. The radiation biology of boron neutron capture therapy. Radiat Res. 1999;151(1):1-18. 